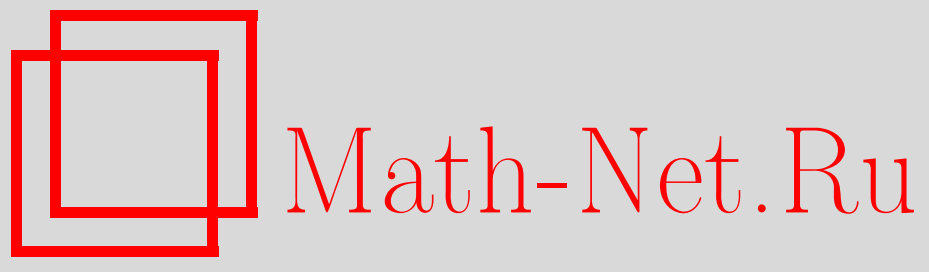

А. А. Гайфуллин, Реализация циклов асферичными многообразиями, УМH, 2008, том 63, выпуск 3, 157-158

DOI: https://doi.org/10.4213/rm9198

Использование Общероссийского математического портала Math-Net.Ru подразумевает, что вы прочитали и согласны с пользовательским соглашением http://www . mathnet.ru/rus/agreement

Параметры загрузки:

IP : 18.209 .158 .208

26 апреля 2023 г., $17: 31: 50$

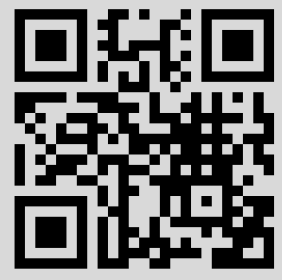




\section{Реализация циклов асферичными многообразиями}

\section{А. А. Гайфуллин}

В конце 1940-х годов Н. Стинрод поставил следующую проблему, известную как проблема о реализации циклов: существуют ли для данного класса гомологий $z \in$ $H_{n}(X ; \mathbb{Z})$ ориентированное многообразие $N^{n}$ и отображение $f: N^{n} \rightarrow X$ такие, что $f_{*}\left[N^{n}\right]=z$ ? Знаменитая теорема Р. Тома утверждает, что с некоторой кратностью каждый целочисленный класс гомологий реализуем по Стинроду. Классической является задача о реализации циклов образами сфер, т. е. задача об изучении образа гомоморфизма Гуревича. В такой постановке не каждый класс гомологий реализуем с некоторой кратностью. Представляет интерес задача о нахождении класса $\mathscr{M}_{n}$ гладких $n$-мерных многообразий, достаточного для реализации с некоторой кратностью всех целочисленных $n$-мерных классов гомологий любого пространства $X$. Центральным результатом настоящей заметки является следующая теорема.

Теорема. Пусть $M^{n}$ - многообразие изоспектральных вещественных симметрических трехдиагональных $(n+1) \times(n+1)$-матрии, и $X$ - произвольное линейно свлзное топологическое пространство. Для любого класса гомологий $z \in H_{n}(X ; \mathbb{Z})$ существуют связное конечнолистное накрытие $p: \widehat{M}^{n} \rightarrow M^{n}$ и отображение $f$ : $\widehat{M}^{n} \rightarrow X$ такие, что $f_{*}\left[\widehat{M}^{n}\right]=q z$ для некоторого челого числа $q$.

К. Томеи [1] доказал, что $M^{n}$ - асферичное гладкое ориентируемое многообразие. (Многообразие называется асферичным, если оно имеет гомотопический тип $K(\pi, 1)$.) Группа $\pi_{1}\left(M^{n}\right)$ была вычислена М. Дэвисом [2]. Он доказал, что она изоморфна свободной от кручения подгруппе конечного индекса группы Кокстера

$$
\begin{gathered}
W=\left\langle s_{1}, \ldots, s_{n}, r_{1}, \ldots, r_{n}\right| s_{i}^{2}=r_{i}^{2}=1, s_{i} s_{j}=s_{j} s_{i} \text { при }|i-j|>1, \\
\left.s_{i} s_{i+1} s_{i}=s_{i+1} s_{i} s_{i+1}, r_{i} r_{j}=r_{j} r_{i}, s_{i} r_{j}=r_{j} s_{i} \text { при } i \neq j\right\rangle .
\end{gathered}
$$

СледСтвиЕ. Каждый целочисленный класс гомологий любого линейно связного пространства может быть с некоторой кратностъю реализован образом ориентированного асферичного гладкого многообразия, фундаменталъная группа которого изоморфна свободной от кручения подгруппе конечного индекса группы $W$.

Положим $[n+1]=\{1,2, \ldots, n+1\}$. Пусть $\mathscr{S}$ - множество всех непустых собственных подмножеств $\omega \subset[n+1]$. Пермутаэдром $\Pi^{n}$ называется выпуклая оболочка точек, полученных всевозможными перестановками координат точки $(1,2, \ldots, n+1) \in$ $\mathbb{R}^{n+1}$. Пермутаэдр является $n$-мерным простым выпуклым многогранником. Его гиперграни $F_{\omega}$ находятся во взаимно однозначном соответствии с подмножествами $\omega \in \mathscr{S}$. Гиперграни $F_{\omega_{1}}$ и $F_{\omega_{2}}$ пересекаются тогда и только тогда, когда $\omega_{1} \subset \omega_{2}$ или $\omega_{2} \subset \omega_{1}$. Каждая грань пермутаэдра $\Pi^{n}$ имеет вид $F_{\omega_{1}} \cap \cdots \cap F_{\omega_{k}}$, где $\varnothing \varsubsetneqq \omega_{1} \varsubsetneqq \cdots \varsubsetneqq$ $\omega_{k} \varsubsetneqq[n+1]$; ее барицентр мы обозначим через $b_{\omega_{1}, \ldots, \omega_{k}}\left(\Pi^{n}\right)$.

K. Томеи [1] построил разбиение многообразия $M^{n}$ на $2^{n}$ пермутаэдров, имеющее вид $M^{n}=\left(\mathbb{Z}_{2}^{n} \times \Pi^{n}\right) / \sim$, где отношение эквивалентности $\sim$ порождено отождествлениями $(g, x) \sim\left(e_{|\omega|} g, x\right)$, если $x \in F_{\omega}$. Здесь $e_{1}, \ldots, e_{n}$ - образующие группы $\mathbb{Z}_{2}^{n}$. Пусть $[g, x]$ - класс эквивалентности пары $(g, x)$. Дадим явную конструкцию разбиения многообразия $\widehat{M}^{n}$ на пермутаэдры, накрывающего разбиение многообразия $M^{n}$. Каждый класс гомологий линейно связного пространства может быть реализован образом сильно связного ориентированного псевдомногообразия $Z^{n}$ (см. определение в $[3 ; \S 24])$. Поэтому нам достаточно доказать теорему в случае $X=Z^{n}$ и $z=\left[Z^{n}\right]$.

Работа выполнена при поддержке РФФИ (грант № 06-01-72551-НЦНИЛ-а) и программы "Ведущие научные школы" (грант НШ-1824.2008.1). 
Перейдя к первому барицентрическому подразделению комплекса $Z^{n}$, мы можем считать, что его вершины окрашены в цвета из множества $[n+1]$ правильным образом, т. е. так, что вершины, соединенные ребром, окрашены в разные цвета. Обозначим через $\mu(\sigma)$ множество цветов вершин симплекса $\sigma$. Для $n$-мерного симплекса $\sigma$ обозначим через $b_{\omega}(\sigma)$ барицентр грани $\tau \subset \sigma$ такой, что $\mu(\tau)=\omega$.

Обозначим через $U$ множество $n$-мерных симплексов комплекса $Z^{n}$. Из наличия правильной раскраски вершин следует, что множество $U$ может быть разбито на две части $U_{+} \sqcup U_{-}$так, что симплексы, имеющие общую гипергрань, лежат в разных частях. Для любого $\omega \in \mathscr{S}$ обозначим через $\mathscr{P}_{\omega}$ множество инволюций $\Lambda: U \rightarrow U$ таких, что $\Lambda\left(U_{ \pm}\right)=U_{\mp}$ и $\mu(\sigma \cap \Lambda(\sigma)) \supset \omega$ для любого симплекса $\sigma \in U$. Множества $\mathscr{P}_{\omega}$ непусты. Определим гомоморфизм $\eta: \mathbb{Z}_{2}^{n} \rightarrow \mathbb{Z}_{2}$ на образующих по формуле $\eta\left(e_{i}\right)=-1$. Определим множество $V$ и инволюции $\Phi_{\omega}: V \rightarrow V$ по формулам

$$
\begin{gathered}
V=\left(U_{+} \times \prod_{\omega \in \mathscr{S}} \mathscr{P}_{\omega} \times \eta^{-1}(1)\right) \cup\left(U_{-} \times \prod_{\omega \in \mathscr{S}} \mathscr{P}_{\omega} \times \eta^{-1}(-1)\right) \subset U \times \prod_{\omega \in \mathscr{S}} \mathscr{P}_{\omega} \times \mathbb{Z}_{2}^{n}, \\
\Phi_{\omega}\left(\sigma,\left(\Lambda_{\gamma}\right)_{\gamma \in \mathscr{S}}, g\right)=\left(\Lambda_{\omega}(\sigma),\left(\widetilde{\Lambda}_{\gamma}\right)_{\left.\gamma \in \mathscr{S}, e_{|\omega|} g\right),}\right.
\end{gathered}
$$

где $\widetilde{\Lambda}_{\gamma}=\Lambda_{\omega} \circ \Lambda_{\gamma} \circ \Lambda_{\omega}$, если $\gamma \subset \omega$, и $\widetilde{\Lambda}_{\gamma}=\Lambda_{\gamma}$, если $\gamma \not \subset \omega$.

Положим $\widehat{M}^{n}=V \times \Pi^{n} / \sim$, где отношение эквивалентности $\sim$ порождено отождествлениями $(v, x) \sim\left(\Phi_{\omega}(v), x\right)$, если $x \in F_{\omega}$.

Проекция $p: \widehat{M}^{n} \rightarrow M^{n}$ определяется по формуле $p\left(\left[\left(\sigma,\left(\Lambda_{\omega}\right)_{\omega \in \mathscr{S}}, g\right), x\right]\right)=[g, x]$. Отображение $p$ корректно определено и является конечнолистным накрытием. Таким образом, $\widehat{M}^{n}$ - гладкое ориентированное многообразие.

Пусть $K$ - триангуляция многообразия $\widehat{M}^{n}$, являющаяся барицентрическим подразделением построенного разбиения на пермутаэдры. Определим отображение $f$ : $\widehat{M}^{n} \rightarrow Z^{n}$ на вершинах триангуляции $K$ по формуле $f\left(\left[v, b_{\omega_{1}, \ldots, \omega_{k}}\left(\Pi^{n}\right)\right]\right)=b_{\omega_{1}}(\sigma)$ и продолжим его по линейности на каждый симплекс триангуляции $K$. Отображение $f$ корректно определено и $f_{*}\left[\widehat{M}^{n}\right]=q\left[Z^{n}\right]$, где $q=2^{n-1} \prod_{\omega \in \mathscr{S}}\left|\mathscr{P}_{\omega}\right|$.

Построенное многообразие $\widehat{M}^{n}$ не обязано быть связным. Искомым многообразием является произвольная связная компонента многообразия $\widehat{M}^{n}$.

Многообразие $\widehat{M}^{n}$ неявно возникало в работе автора [4] в частном случае более общей конструкции реализации циклов. Разбиение многообразия $\widehat{M}^{n}$, построенное в настоящей работе, отлично от разбиения, построенного в [4], и приспособлено для доказательства того, что $\widehat{M}^{n}$ накрывает $M^{n}$.

Автор благодарен В. М. Бухштаберу за постановку задачи и постоянное внимание, а также С. М. Натанзону, А. В. Пенскому, А.Б. Сосинскому и О. В. Шварцману за полезные обсуждения.

\section{Список литературы}

[1] C. Tomei, Duke Math. J., 51:4 (1984), 981-996. [2] M. W. Davis, Duke Math. J., 55:1 (1987), 105-139. [3] Г. Зейферт, В. Трельфалль, Топология, Л., 1938; пер. с нем.: H. Seifert, W. Threlfall, Lehrbuch der Topologie, Teubner, Leipzig-Berlin, 1934. [4] А. А. Гайфуллин, УМН, 62:6 (2007), 167-168; англ. пер.: A. A. Gaifullin, Russian Math. Surveys, 62:6 (2007), 1199-1201.

\section{A. А. Гайфуллин (А. А. Gaifullin)}

Московский государственный университет им. М. В. Ломоносова

E-mail: gaifull@mccme.ru
Представлено В. М. Бухштабером Принято редколлегией 19.03.2008 